\title{
Controlling Magnetization Reversal and Hyperthermia Efficiency in Core-Shell Iron-Iron Oxide Magnetic Nanoparticles by Tuning the Interphase Coupling
}

\author{
K. Simeonidis, C. Martinez-Boubeta, D. Serantes,* S. Ruta, O. Chubykalo-Fesenko, R. Chantrell, \\ J. Oró-Solé, Ll. Balcells, A. S. Kamzin, R. A. Nazipov, A. Makridis, and M. Angelakeris*
}

Cite This: ACS Appl. Nano Mater. 2020, 3, 4465-4476

Read Online

ACCESS | Lلll Metrics \& More | 回 Article Recommendations ｜ sl Supporting Information

ABSTRACT: Magnetic particle hyperthermia, in which colloidal nanostructures are exposed to an alternating magnetic field, is a promising approach to cancer therapy. Unfortunately, the clinical efficacy of hyperthermia has not yet been optimized. Consequently, routes to improve magnetic particle hyperthermia, such as designing hybrid structures comprised of different phase materials, are actively pursued. Here, we demonstrate enhanced hyperthermia efficiency in relatively large spherical $\mathrm{Fe} / \mathrm{Fe}$-oxide core-shell nanoparticles through the manipulation of interactions between the core and shell phases. Experimental results on representative samples with diameters in the range $30-80 \mathrm{~nm}$ indicate a direct correlation of hysteresis losses to the observed heating with a maximum efficiency of around $0.9 \mathrm{~kW} / \mathrm{g}$. The absolute particle size, the core-shell ratio, and the interposition of a thin wüstite interlayer are shown to have powerful effects on the specific absorption rate. By comparing our measurements to micromagnetic calculations, we have unveiled the occurrence of topologically nontrivial magnetization reversal modes under which interparticle interactions become negligible, aggregates formation is minimized and the energy that is converted into heat is increased. This information has been overlooked until date and is in stark contrast to the existing knowledge on homogeneous particles.

KEYWORDS: iron, iron oxides, magnetic hyperthermia, core-shell nanoparticles, hybrid nanostructures

\section{INTRODUCTION}

The capability of magnetic nanoparticles to convert radiofrequency (RF) electromagnetic energy into heat has drawn a strong and growing research interest during the past decade, with examples in the field of catalysis, ${ }^{1}$ lightweight thermoplastic composites for aeronautical and automotive engineering, $^{2}$ and numerous biomedical applications. ${ }^{3,4}$ In particular, biocompatible magnetic nanoparticles have emerged as promising agents for use in drug delivery ${ }^{5}$ and selective destruction of tumors by hyperthermia. ${ }^{6}$ Numerous clinical and basic studies have shown that temperature stresses can alter tumor endurance in a significant manner. The use of RF fields instead of conventional methods allows for minimally invasive and real-time control of the temperature, particularly in deep body regions, while preventing damage to healthy tissues. At present, many fundamental research studies have been carried out worldwide and some have entered into clinical trials, especially when magnetic hyperthermia is combined with more traditional therapeutic approaches, such as the codelivery of anticancer drugs or radiation therapy.

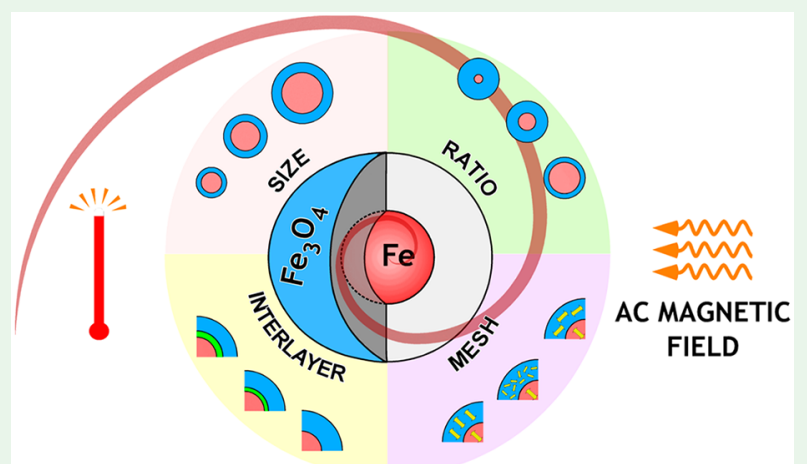

Despite rapid progress, the precise way to increase the heating efficacy of magnetic nanoparticle-based therapeutics is unknown. ${ }^{8}$ This has posed a challenge for theoretical modeling. In fact, most of the reported hyperthermia data as a function of particle concentration is encompassed by a universal response. ${ }^{9}$ On the one hand, manipulation of the field conditions is the primary source of efficiency control. ${ }^{10,11}$ Importantly, recent numerical analyses carried out by using 3D realistic models of the human body indicated that acceptable values for the magnetic field amplitude/frequency product $(H$. $f$ ) may increase up to four times in comparison to the usually considered safety threshold of $\sim 5 \times 10^{8} \mathrm{~A} / \mathrm{ms}^{12}$ On the other hand, factors, such as the nanoparticle features (composition, size, shape), their geometrical arrangement and volume

Received: February 27, 2020

Accepted: April 13, 2020

Published: April 13, 2020

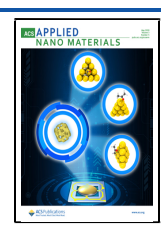


Scheme 1. Overview of Core-Shell Nanoparticles Production Methodology, Growth Mechanism, and Function as Magnetic Hyperthermia Agents with Tunable Magnetic Reversal Potential

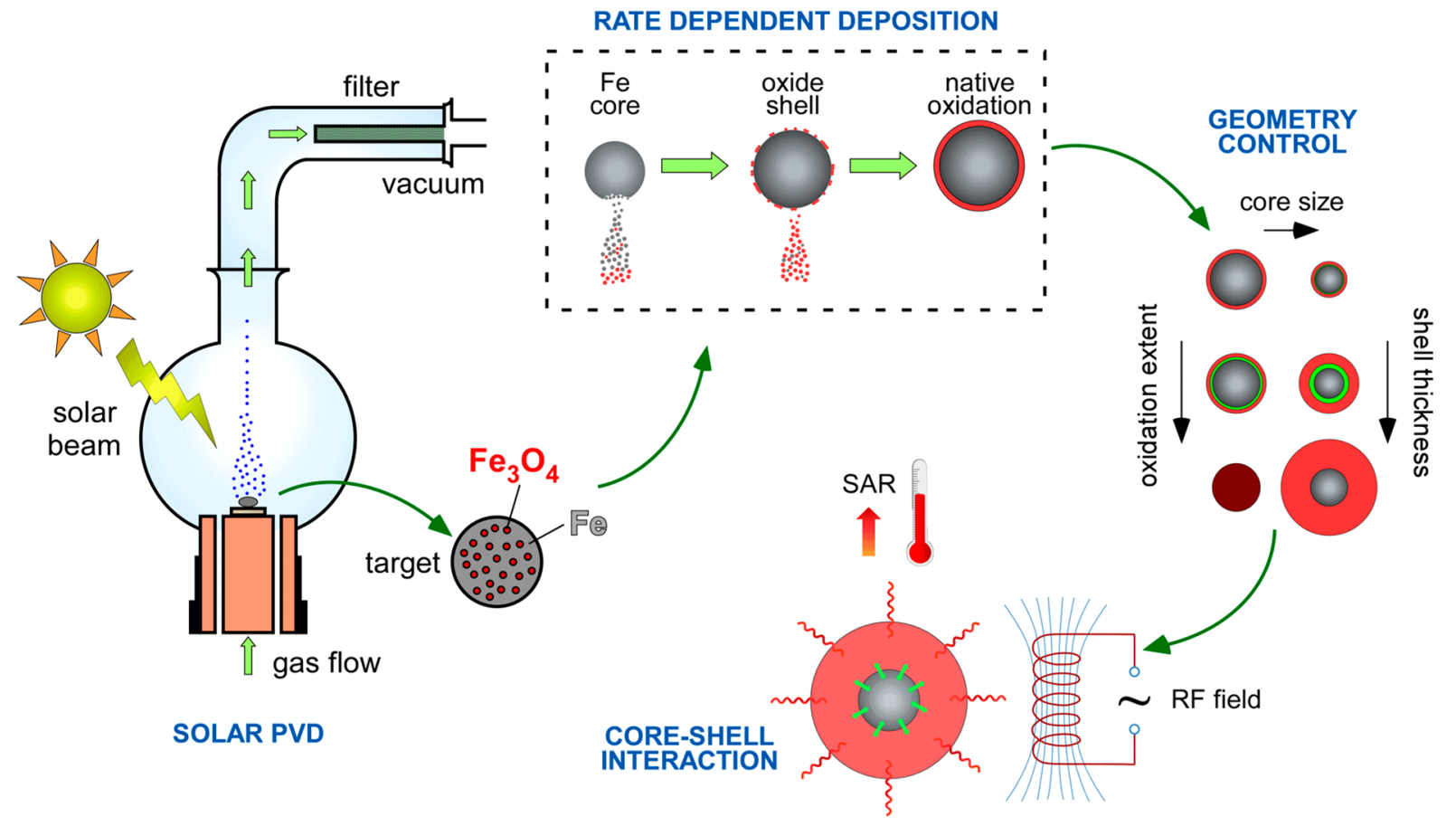

concentration within the dielectric cellular matrix also play an important role. ${ }^{13}$ And so, in practice, the heat transfer from magnetic nanoparticles subjected to an oscillating field is not only affected by their intrinsic properties but also by the surrounding environment. For instance, the thermal conductivity of nanofluids is largely dependent on whether the nanoparticles stay dispersed, form large aggregates, or assume a percolating linear configuration. ${ }^{14}$

Minimizing the dosage of nanoparticles required for an effective treatment is another task of high significance. In that respect, ferrite nanoparticles are undoubtedly the most convenient building blocks for magnetic hyperthermia because of their good biocompatibility and stability. But conventional single-phase iron oxide systems, and especially, those with smaller dimensions, where Brown mechanisms for heating are practically disabled during particles immobilization in biological environments, ${ }^{15}$ suffer from low heating efficiency. Any further improvement of their known performance would involve precise chemical engineering on the nanoscale. ${ }^{6,16}$ Here, we present results of an experimental study that directly relates the structural and magnetic properties of iron-based core-shell particles. In particular, it is shown that surrounding a ferromagnetic (FM) iron core with a ferrimagnetic iron oxide shell can tune the specific absorption rate (SAR) to unprecedented efficiency (Scheme 1). Additional modeling demonstrates that different reversal mechanisms are promoted, depending on the core-shell ratio and absolute particle size. More crucial to the synchronized behavior of this multiphase material, we find that the presence (or not) of a nonmagnetic interlayer, which changes the interphase coupling and alters the reversal mechanism, is key to allowing for diminished importance of the detrimental interparticle dipolar interactions. This constitutes a remarkably different approach in comparison with other works dealing with core-shell geometries in which the objective was essentially to tune the effective single-particle properties but still under the giant-spin (i.e., coherent rotation of the nanoparticle unit) hypothesis. ${ }^{17,18}$ This scenario opens the way to a superior control of the heating performance, particularly for in vivo applications, where it is known to attenuate in comparison to in vitro measurements.

\section{EXPERIMENTAL SECTION}

Particles' Preparation. A very simple, fast, green, and costeffective way by using solar vapor phase condensation was used. This technique allows the preparation of large quantities of nanoparticle dry powders presenting a narrow particle size distribution without purification steps. ${ }^{19}$ Families of particles were prepared using almost all $3 \mathrm{~d}$ metals and many others, but here, we use iron, which is considered to be an essential nutrient for cells that are dividing rapidly, such as in tumors, and also plays a vital role to perform various body functions. Moreover, produced iron nanoparticles are featured with some significant advantages very difficult to be delivered by wet chemistry methods for nanoparticles preparation: chemical stability over a prolonged period of time without the need for special handling, low surface charge, and energy resulting in weak aggregation tendency. Particularly, evaporation of pure Fe powder pressurized in a pellet form, under Ar flow (70 Torr) resulted in $50 \mathrm{~nm}$ nanoparticles. Under the same pressure, coevaporation of $\mathrm{Fe}$ and $\mathrm{Fe}_{3} \mathrm{O}_{4}$ mixtures produces a similar particle diameter but the thickness of oxide on the surface is anticipated to increase as compared to the shell stabilized after natural oxidation. The decrease in particles diameter (down to about $35 \mathrm{~nm}$ ) was achieved by using lower pressure (50 Torr), whereas evaporation of a pure $\mathrm{Fe}_{3} \mathrm{O}_{4}$ target at 80 Torr enables the production of larger nanoparticles $(75 \mathrm{~nm})$ with a very low zerovalent $\mathrm{Fe}$ content. The pumping of oxygen is sufficient to provide a completely oxidized dark-red product $\left(\gamma-\mathrm{Fe}_{2} \mathrm{O}_{3}\right)$. All samples were stored in a desiccator periodically pumped with nitrogen to succeed the extension of the time-scale without significant surface oxidation to a many months period.

Morphology and Crystal Structure. Structural characterization was performed by X-ray diffraction (XRD) using a Rigaku Ultima+ powder diffractometer with $\mathrm{Cu} \mathrm{K}_{\alpha}$ radiation. Transmission electron microscopy (TEM) images and selected area electron diffraction (SAED) patterns were obtained on a JEOL JEM-1210 operating at $120 \mathrm{kV}$. A number of samples were examined in more detail using 
Table 1. Summary of the Parameters Used for the Simulations

\begin{tabular}{lcclc} 
& $M_{\mathrm{S}}\left(\mathrm{emu} / \mathrm{cm}^{3}\right)$ & $K_{\mathrm{C}}\left(\mathrm{erg} / \mathrm{cm}^{3}\right)$ & $A_{\text {exch }}(\mathrm{J} / \mathrm{m})$ & $2.5 \times 10^{-11}$ \\
$\mathrm{Fe}$ core & 1711 & $4.6 \times 10^{5}$ & $1.0 \times 10^{-11}$ \\
$\mathrm{Fe}_{3} \mathrm{O}_{4}$ shell & 477 & $-1.1 \times 10^{5}$ & 0 \\
$\mathrm{FeO}$ spacer & 1 & 0 & $0,1,0)$ \\
\hline
\end{tabular}

Table 2. Overview of Preparation Conditions, Main Characteristics, and Schematic Illustrations of Studied Samples ${ }^{a}$

\begin{tabular}{|c|c|c|c|c|c|c|c|c|c|c|}
\hline \multirow{3}{*}{ Sample } & \multirow{2}{*}{\multicolumn{2}{|c|}{ Target (\% wt.) }} & \multirow{3}{*}{$\begin{array}{c}\text { Chamber's } \\
\text { pressure } \\
\text { (torr)/Gas }\end{array}$} & \multicolumn{4}{|c|}{ Composition (\% wt.) } & \multirow{3}{*}{$\begin{array}{l}\text { Average } \\
\text { size }(\mathrm{nm})\end{array}$} & \multirow{2}{*}{\multicolumn{2}{|c|}{ Morphology* }} \\
\hline & & & & \multicolumn{4}{|c|}{ XRD/(Mossbauer analysis)* } & & & \\
\hline & $\mathbf{F e}$ & $\begin{array}{c}\mathrm{Fe}_{3} \\
\mathrm{O}_{4}\end{array}$ & & $\mathbf{F e}$ & $\mathrm{FeO}$ & $\mathrm{Fe}_{3} \mathrm{O}_{4}$ & $\begin{array}{c}\gamma- \\
\mathrm{Fe}_{2} \mathrm{O}_{3}\end{array}$ & & $\begin{array}{l}\text { Core- } \\
\text { shell(s) } \\
(\mathrm{nm})\end{array}$ & Scheme \\
\hline F01 & 100 & - & 70/Ar & $\begin{array}{r}79.4 \\
(77.7)\end{array}$ & - & $\begin{array}{c}20.6 \\
(22.3)\end{array}$ & - & 50 & $44 / 3$ & \\
\hline F02 & 75 & 25 & 70/Ar & $\begin{array}{c}58.6 \\
(69.8)\end{array}$ & $\begin{array}{l}10.1 \\
(5.2)\end{array}$ & $\begin{array}{c}31.3 \\
(25.0)\end{array}$ & - & 52 & $42 / 2 / 3$ & \\
\hline F03 & 75 & 25 & $50 / \mathrm{Ar}$ & $\begin{array}{r}53.6 \\
(67.5)\end{array}$ & $\begin{array}{c}11.8 \\
(13.1)\end{array}$ & $\begin{array}{c}34.6 \\
(-)\end{array}$ & $\begin{array}{c}- \\
(19.3)\end{array}$ & 33 & $25 / 1 / 3$ & \\
\hline F04 & 50 & 50 & 70/Ar & $\begin{array}{c}12.9 \\
(11.1)\end{array}$ & $\begin{array}{l}14.7 \\
(8.4)\end{array}$ & $\begin{array}{c}72.4 \\
(80.6)\end{array}$ & - & 48 & $24 / 4 / 8$ & \\
\hline F05 & - & 100 & $80 / \mathrm{Ar}$ & $\begin{array}{c}9.1 \\
(5.9)\end{array}$ & - & $\begin{array}{l}90.9 \\
(94.1)\end{array}$ & - & 78 & $30 / 24$ & \\
\hline F06 & - & 100 & $80 / \mathrm{Ar}-\mathrm{O}_{2}$ & - & $\begin{array}{c}- \\
(4.6)\end{array}$ & $\begin{array}{c}- \\
(58.0)\end{array}$ & $\begin{array}{c}100 \\
(37.4)\end{array}$ & 43 & $43 /-$ & \\
\hline
\end{tabular}

${ }^{a}$ Single asterisk $(*)$, estimated by geometrical features, compositional analysis, and magnetic properties; double asterisk (**), composition analysis by Mossbauer spectroscopy is indicated into parentheses.

high-resolution (HRTEM) and scanning transmission electron (STEM) microscopy with a FEI Tecnai G2 F20 microscope operating at $200 \mathrm{kV}$ and equipped with EDAX element analysis system. Samples were prepared by dispersing the powders in ethanol. A small droplet of the suspension was placed on a holey carbon film supported on a copper grid. Scanning electron microscopy (SEM) micrographs of numerous samples were obtained in a Quanta 200 ESEM FEG FEI microscope.

Mössbauer Spectroscopy. To give more accurate description of coexisting iron phases, the powders were investigated by ${ }^{57} \mathrm{Fe}$ Mössbauer spectrometry. The spectra were collected at room temperature in transmission geometry, using a conventional constant acceleration spectrometer operating in triangular wave mode and a ${ }^{57}$ Co source into rhodium matrix. The speed scale of the Doppler modulator and isomer shift calibration were performed using a $10 \mu \mathrm{m}$ thick $\alpha$-Fe foil. Mössbauer spectra were fitted using the Mosfit software, a least-squares iteration program. ${ }^{20}$ Hyperfine interaction parameters are denoted as follows: IS for isomer shift $(\mathrm{mm} / \mathrm{s})$, QS for quadrupolar shift $(\mathrm{mm} / \mathrm{s})$, and $H_{\text {eff }}$ for magnetic hyperfine field $(\mathrm{T})$. Estimated errors $( \pm 5 \%)$ originate from the statistical inaccuracy given by the fitting program. Results concerning composition were compared and validated to the quantified data from the corresponding XRD diagrams using the Rietveld methodology.

Magnetism. Magnetic features of fixed nanoparticles powder were evaluated by room temperature vibrating sample magnetometer (VSM-1.2H/CF/HT Oxford Instruments) in a magnetic field range $\pm 1 \mathrm{~T}$. Additional minor hysteresis loops, carried out at maximum fields equal to the calorimetric magnetic hyperthermia measurements $(30$ and $60 \mathrm{mT}$ ), were also recorded after demagnetizing the samples to determine the maximum attainable power loss that can be generated by each system. Quantitative evaluation of DC minor loops appears as a very reliable way to get a first estimation of the potential of each sample to provide hysteresis loss heat dissipation in cases where hysteresis represents the dominant magnetic heating mechanism. However, it should not be confused with the actual behavior of the magnetic nanoparticles under the high frequency hyperthermia field.

Calorimetric Measurements. The heating efficiency of nanoparticles dispersions in distilled water was evaluated in two different devices using a higher frequency $(765 \mathrm{kHz})$ AC magnetic field peak of $30 \mathrm{mT}$ and a lower frequency $(210 \mathrm{kHz})$ setup capable of operating in 30-60 $\mathrm{mT}$ field amplitude range. Temperature was monitored by using a GaAs-based fiber optic probe immersed in a test tube 
containing $1 \mathrm{~mL}$ of dispersion. The quantifiable index of heating efficiency, the specific absorption rate or specific loss power, was derived from the slope of the temperature versus time curve in nonadiabatic conditions with detailed modeling of the heat exchange with the surrounding environment. ${ }^{21-23}$ Despite reduced dipolar interactions in the studied samples, the application of the AC field during the hyperthermia experiment was sufficient to cause a gradual precipitation of the solid within the first $100 \mathrm{~s}$ of measurement. After this time, nanoparticles were arranged at the side of the vessel forming a ring next to the induction coil. This effect is unavoidable in any magnetic nanoparticles system; however, recorded heating curves did not seem to be influenced by this inhomogeneity transition and this is probably attributed to the high heat transfer rate from nanoparticles to the whole solution volume.

Computational Details. Micromagnetic simulations were carried out using the $3 \mathrm{D}$ version of the OOMMF package. ${ }^{24}$ In the first approximation, all particles were assumed spherical with a singlecrystal core. $M(H)$ hysteresis loops were simulated for several coreshell structures varying the $\mathrm{Fe}_{3} \mathrm{O}_{4}$ shell thickness-to-Fe core radius, that is, $t_{\mathrm{S}} / r_{\mathrm{C}}$ ratio. When necessary, a separating $\mathrm{FeO}$ interlayer of width $t_{\mathrm{sp}}$ was included. In all cases we considered a $1 \mathrm{~nm}$-side cubic cell discretization, that is, below the smaller specific exchange length, $l_{\text {exch1 }}=3.7 \mathrm{~nm}$ for the iron core and $l_{\text {exch1 }}=8.4 \mathrm{~nm}$ for the $\mathrm{Fe}_{3} \mathrm{O}_{4}$ shell. Field was set collinear to the [100] easy axis of magnetization of iron (as corresponds to physically oriented nanoparticles under applied field in viscous conditions). ${ }^{25}$ No thermal excitations were considered, thus rendering the results applicable only to blocked nanoparticles (reasonable assumption for the large sizes considered ${ }^{26,27}$ ). The micromagnetic parameters are shown in Table 1 , with the exchange between core and shell assumed to be $0.175 \times 10^{-11} \mathrm{~J} / \mathrm{m}$ (i.e., about $10 \%$ of the average between the two phases). The easy axes of the shell are randomly oriented. The separating spacer is treated as paramagnetic-like, with a negligible $M_{S}$ value, that is, easily polarized and with no magnetic exchange or anisotropy contributions. The simulations were performed assuming quasi-static conditions (reasonable for FM-like behavior, as discussed elsewhere ${ }^{9,28}$ ). To discard undesired artificial effects of the initial configuration for minor loops, the results were checked against the initialization in the random or vortex states.

\section{RESULTS AND DISCUSSION}

Structure, Composition, and Magnetism. Compositional tuning is a major strategy to tailor magnetic properties. To this end, we have fabricated a series of complex structures using solar vapor phase condensation by varying the Fe-to$\mathrm{Fe}_{3} \mathrm{O}_{4}$ target ratio, the chamber pressure and the oxidizing conditions as defined by pumping gas. Briefly, the evaporation of $\mathrm{Fe}$ under inert conditions preserves the metallic characteristics in a core that is lately passivated with a thin oxide shell, while simultaneous $\mathrm{Fe}_{3} \mathrm{O}_{4}$ decomposition also produces a small metallic core but surrounded by a thicker oxide shell. Moreover, depending on the composition of the gas stream during synthesis (Ar or air), completely oxidized nanoparticles $\left(\gamma-\mathrm{Fe}_{2} \mathrm{O}_{3}\right)$ may also be produced. ${ }^{22}$ Table 2 shows a compilation of both the preparation conditions and structural and morphological results extracted from a compendium of characterization techniques. Figure S1 (see Supporting Information) presents SEM images of samples under study.

In general, our experimental system is a nanocrystalline magnetic sphere composed of several layers (up to three) of $\mathrm{Fe}$ and its oxides. The initial structural analysis of obtained nanopowders was performed by XRD diagrams (see Figures S2 and S3). A first set of samples (F01-02) includes a Fe grain size about $40 \mathrm{~nm}$ surrounded by variable oxides $\sim 5 \mathrm{~nm}$ thickness. Results suggest that, when a metallic target is evaporated under inert gas flow (sample F01), a large percentage of $\mathrm{Fe}$ (up to $80 \% \mathrm{wt}$.) is preserved in the prepared
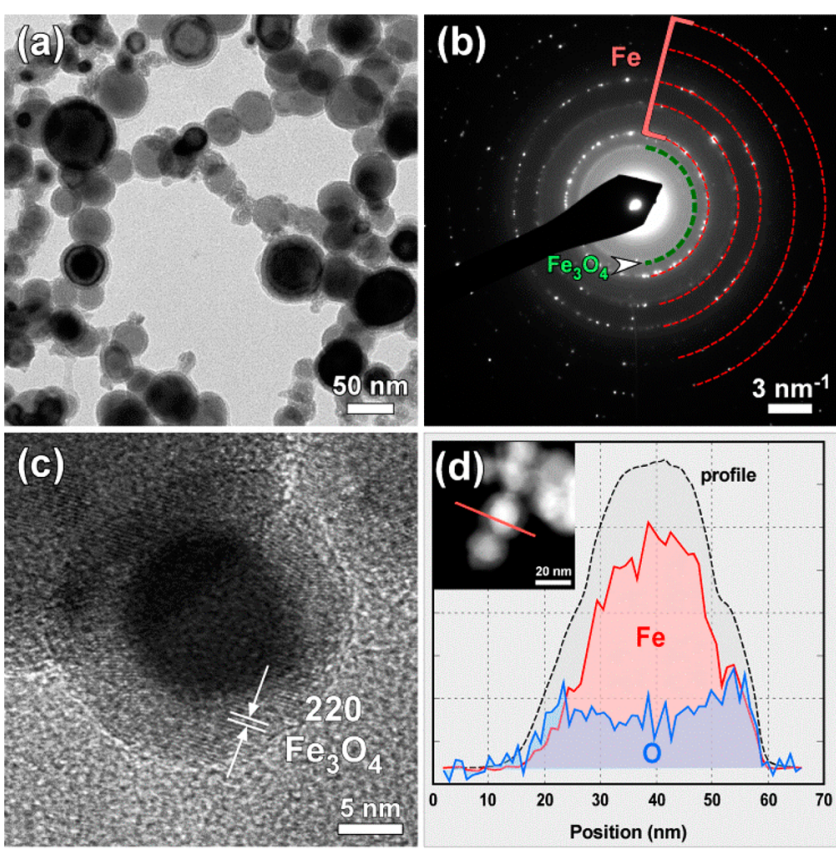

Figure 1. Representative TEM image of sample F03 (a) and corresponding selected area electron diffraction pattern (b) showing coexistence of metallic iron (red rings) and magnetite (green ring). Focusing on a small particle, HRTEM discloses a fluffy coating layer of magnetite (c). STEM mode imaging and composition profile derived from energy-dispersive spectroscopy analysis demonstrating the iron-rich core (d).

nanoparticles while the surrounding outer shell is probably related to native oxidation. Another series (F03-05) comprises a constant $\mathrm{Fe}$ core (25 $\mathrm{nm}$ in diameter) coated by oxide shells with increasing thicknesses from 4 up to $24 \mathrm{~nm}$. By introducing $\mathrm{Fe}_{3} \mathrm{O}_{4}$ in the evaporating pellet material, the oxide content gradually increases and becomes the dominant phase when the $\mathrm{Fe} / \mathrm{Fe}_{3} \mathrm{O}_{4}$ ratio in the target reaches 1:1 (sample F04). However, even in the evaporation of pure $\mathrm{Fe}_{3} \mathrm{O}_{4}$, a small percentage of $\mathrm{Fe}$ is still observed as a result of $\mathrm{Fe}_{3} \mathrm{O}_{4}$ decomposition during the evaporation procedure (sample F05). Finally, a pure oxide sample (F06) stands for comparison to the $40 \mathrm{~nm}$ metallic nuclei.

Further insight into the microstructure by using TEM and elemental mapping provides evidence about the core-shell distribution of $\mathrm{Fe}$ and its oxides, depicted in Figure 1, representatively, for the smallest overall diameter (sample F03). Low-resolution images (Figure 1a) clearly show the morphology of the particles and point to the presence of a lower contrast material casing a darker center, but the presence of the core-shell morphology is definitively proven by the high-resolution TEM image of the individual particles. The electron diffraction results (Figure $1 \mathrm{~b}$ ), in consistence to XRD, suggest the coexistence of pure metal and oxide phases. Figure 1c presents a high contrast difference of the core and shell regions where the oxide phase is indexed as $\mathrm{Fe}_{3} \mathrm{O}_{4}$. Further, local electron energy loss spectra analysis across the whole particle (Figure 1d) confirms that element distribution corresponds to the iron core/oxide shell morphology.

We note that, independently of the median size, the relative distribution width is constant at about $15-20 \%$, a characteristic of our synthesis process. ${ }^{22,29}$ Indeed, it is obvious that, in real situations, there are spreads in the volume of particles but also the system may contain a number of different phases, each 


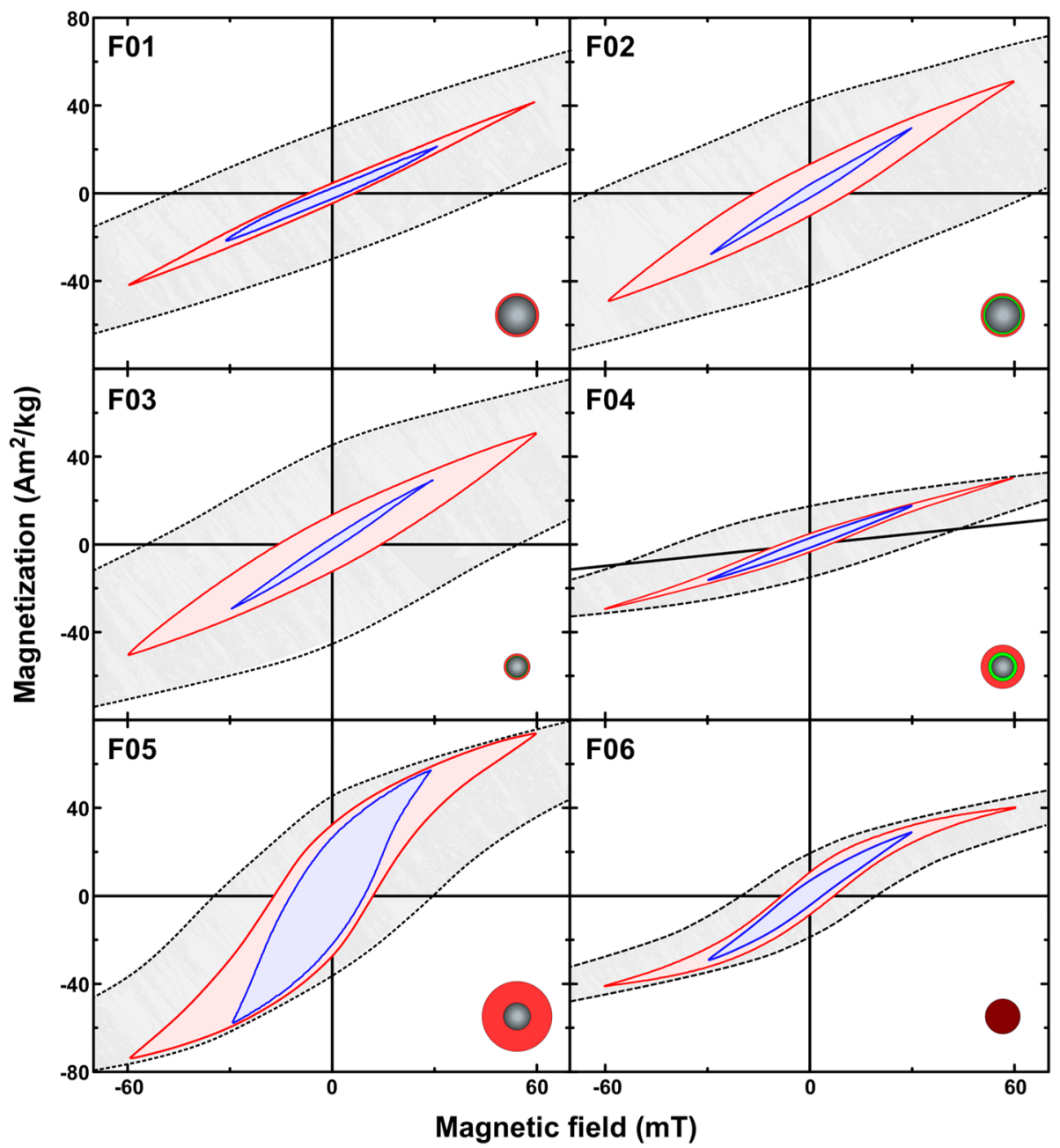

Figure 2. Minor hysteresis loops obtained by cycling with maximum field of 30 (in blue) and 60 (red) mT for samples under study with decreasing Fe content (F01-F06). The gray-shaded region accounts for the corresponding full cycles with $1 \mathrm{~T}$, well above saturation field.

with a specific saturation magnetic moment and anisotropy energy, and, therefore, several relaxation parameters. Mössbauer spectroscopy measures directly the contribution from magnetically distinct phases. At room temperature (see Table S1 and Figure S4 in the Supporting Information), the observed intensity ratio $3: 2: 1$ between the sextet lines and the value of the hyperfine magnetic field $(\sim 33 \mathrm{~T})$ indicate that the core of most of the samples (except F06) is composed of bcc Fe, together with broad oxide components superimposed to the metallic iron. This picture is consistent also with the XRD and electron microscopy analyses.

Within the resolution of the structural analysis, it appears that the magnetic state of the nucleus remains unchanged, no matter the shell. In this regard, the iron oxide phases consist of two components $\left(\mathrm{Fe}_{3} \mathrm{O}_{4}\right.$ and $\mathrm{Fe}_{2} \mathrm{O}_{3}$-like $)$. We and others have demonstrated that any sample made of nanocrystalline ironoxides is a mixture of several stoichiometries, whereas the small particles are maghemite rich. ${ }^{30}$ It is well-established that the octahedral $\mathrm{Fe}^{2+}$ ions located at the surface of magnetite particles are very sensitive to oxidation, ${ }^{31}$ thus giving rise to this $\mathrm{Fe}^{3+}$ rich layer, more visible in the smaller particles (sample F03) and those grown under the oxidizing atmosphere (sample F06). The corresponding oxide phase (mostly $\mathrm{Fe}_{3} \mathrm{O}_{4}$ ) in the case of pure iron targets (sample F01) is explained by the inevitable surface passivation after exposure to ambient conditions. Moreover, every time that the target was a mixture of $\mathrm{Fe}$ and $\mathrm{Fe}_{3} \mathrm{O}_{4}$ in variable proportions (samples F02, F03, F04), the Mössbauer spectrum shows also a broad paramagnetic central peak, which can be assigned to nonstoichiometric wüstite ( $\mathrm{FeO}$ is an antiferromagnet with a Néel temperature around $200 \mathrm{~K}$ ). It is worth mentioning that $\mathrm{FeO}$ is known to disproportionate into $\mathrm{Fe}$ and $\mathrm{Fe}_{3} \mathrm{O}_{4}{ }^{32}$ Giving a model to explain the oxidation process of iron, it seems reasonable that this ferrous $\mathrm{FeO}$ phase would be placed at the core-shell interface, between the pure metallic Fe and the oxidized outer region. ${ }^{33,34}$ Obviously, epitaxial strains arise due to the accommodation of the crystalline lattices of the oxide and the metal. And probably, this strain favors the nucleation of the otherwise metastable FeO-like phase. For example, in thin films, the misfit between the iron atoms in the $[\overline{1} 10]$ direction of $\mathrm{FeO}$ and the [001] direction of iron is approximately 6\%, whereas between $\mathrm{FeO}$ and $\mathrm{Fe}_{3} \mathrm{O}_{4}$ it is only $3 \%$. Regarding the oxidation of iron nanoparticles, there is still little information on the evolution of growth stresses in these multiphase regimes. Naturally, a tensile stress is expected in the $\mathrm{Fe}_{3} \mathrm{O}_{4}$ shell opposing the compressive strain on the core, ${ }^{35}$ and this stress increases, at the very initial phase, with the thickness of the oxide layer. ${ }^{36}$ In our case, stress occurrence as witnessed by XRD main peak shift attributed to reduction in average lattice parameter is reported in Figure S2. 


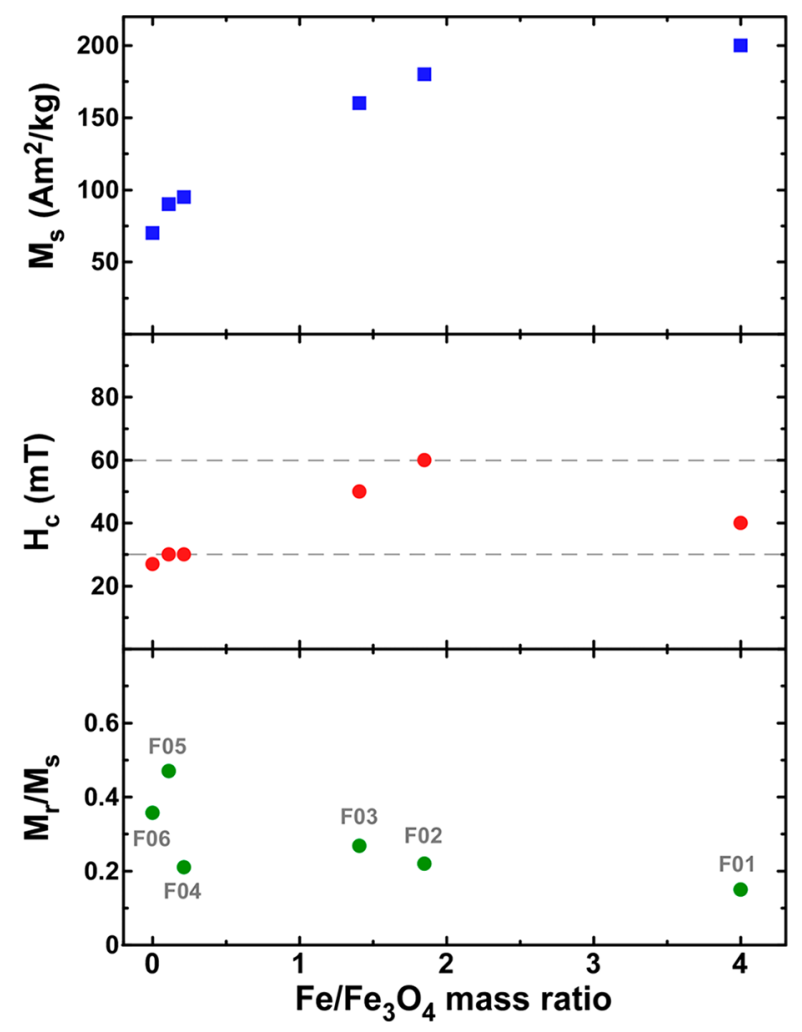

Figure 3. Room-temperature dependence of saturation magnetization, coercive field and remanence ratio as a function of the $\mathrm{Fe} / \mathrm{Fe}_{3} \mathrm{O}_{4}$ mass ratio of core-shell magnetic nanoparticles. Horizontal lines in the $H_{c}$ plot account for maximum fields in minor cycles $(30$ and $60 \mathrm{mT})$ and hyperthermia measurements.

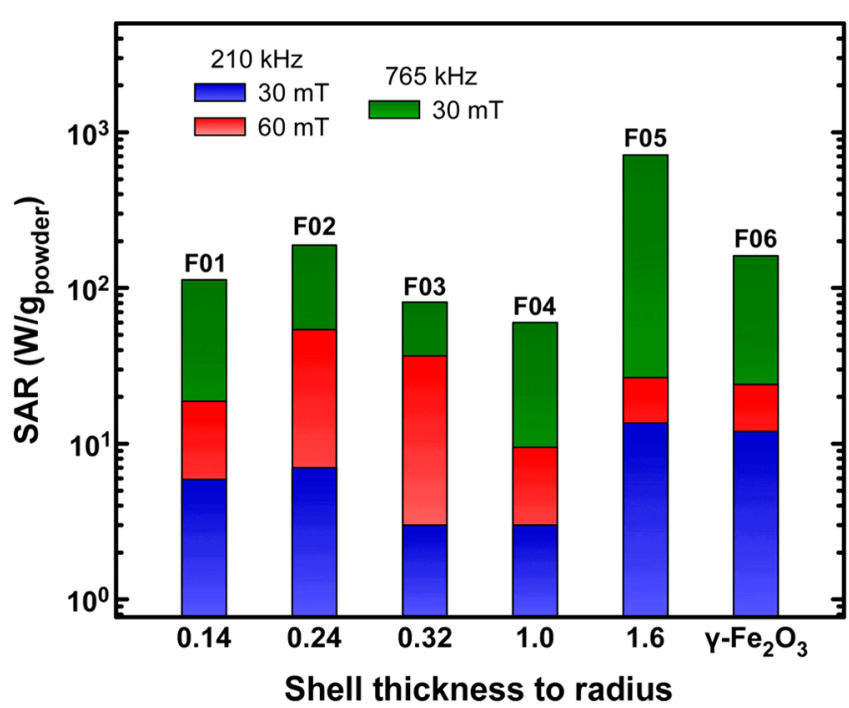

Figure 4. Hyperthermia efficiency for fixed frequency $(210 \mathrm{kHz})$ but different field amplitudes and for the same field amplitude $(30 \mathrm{mT})$ but different AC field frequency $(765 \mathrm{kHz})$. Particles concentration is $4 \mathrm{mg} / \mathrm{mL}$. Sample F06 would correspond to infinite shell/core ratio.

Magnetization measurements shown in Figure 2 demonstrate different reversal mechanisms depending on the coreshell ratio and absolute particle size. On the one hand, the saturation magnetization increases with the percentage of iron and reaches $200 \mathrm{Am}^{2} / \mathrm{kg}$ for F01, which is close to the pure bulk value. But, as shown in Figure 3, this trend is not reflected in monotonic behavior of the $M_{\mathrm{r}} / M_{\mathrm{s}}$ ratio and coercivity as Fe content decreases and oxide percentage is added gradually to the shell. The coercivity first increases, reaches a maximum at a Fe-to-oxide ratio of 2 and then decreases. We highlight that for iron, maximum coercivities because of the magneto-crystalline anisotropy alone are about $40 \mathrm{mT},{ }^{37}$ though strain anisotropies may force it up to $60 \mathrm{mT}$, which is the case reported here. Complete oxidation of nanoparticles (sample F06) results again in weaker magnetic features.

Incidentally, room-temperature magnetic properties of core-shell iron/iron oxide nanocrystals with core sizes ranging from $\sim 5$ to $20 \mathrm{~nm}$ and constant thickness of the oxide shell about $2 \mathrm{~nm}$ were previously investigated by Kaur et al. ${ }^{38}$ They concluded that dipolar interparticle interactions become stronger with the growth of size. There, the decrease in coercive field $H_{\mathrm{c}}$ from $50 \mathrm{mT}$ to about $5 \mathrm{mT}$ with decreasing particle size was due to thermal effects. This is not the case here, and we surmise that not only interparticle but also intraparticle interactions between the iron core (ferromagnetic) and oxide shells should be considered.

So far, we have determined the quasi-static magnetic response in a core-shell iron/iron oxide system. A combined study of microscopy, XRD, and Mössbauer reveals the existence of several oxides in the shell region, with a major contribution from $\mathrm{FeO}$ and $\mathrm{Fe}_{3} \mathrm{O}_{4}$. Apart from the compositions, hysteresis loops demonstrate that optimizing material performance requires the change in core-shell size proportion. Thus, it is anticipated that particular ratios of soft to hard phases would lead to enhanced heating response.

Magnetic Heating. The efficiency of magnetoheating particles is primarily governed by three interrelated parameters: the magnetic anisotropy energy (which depends on several features, for example, the size of the particles via surface effects), the experimental field conditions, and the dosage. Figure 4 indicates that since the amount of generated heat is given by the product of the frequency and the area of the hysteresis loop, SAR is gradually enhanced upon increasing the frequency. Concomitantly, according to the hysteresis losses (see Figure 2), oxide-rich nanoparticles are by far more efficient at lower AC fields (see data at $30 \mathrm{mT}$ ) while Fe-rich samples require higher fields to unfold their full response, as also anticipated from the $H_{\mathrm{c}}$ and $M_{\mathrm{r}}$ dependencies shown in Figure 3. On the other hand, the complete oxidation results in similar response to that for typical iron oxides reported in literature. ${ }^{39}$ Thus, it seems that the change in the two phases ratio can lead to both magnetic hardening and enhanced heating efficiency, provided the proper hyperthermia conditions are applied (AC frequency and field amplitude). Indeed, we acknowledge that similar (bi)magnetic iron/iron oxide core-shell nanoparticles (though $<15 \mathrm{~nm}$ in size) have been already explored for in vivo hyperthermia. ${ }^{40}$ But extraordinary, in our case, sample F05, with oxide shell thickness $t_{\mathrm{S}}=24 \mathrm{~nm}$ and Fe core's radius $r_{\mathrm{C}}=15 \mathrm{~nm}$, shows a remarkable heating efficiency which approaches $0.9 \mathrm{~kW} / \mathrm{g}$. On this, one should remark the puzzling coincidence that the term $\left(t_{\mathrm{S}}+r_{\mathrm{C}}\right) / t_{\mathrm{S}}$ is found practically equal to the term $t_{\mathrm{S}} / r_{\mathrm{C}}$ and both very close to the value of $\varphi=1.618 \ldots$, which is known as the geometric golden ratio. A similar finding has been reported for core-shell bimagnetic $\mathrm{Fe}_{3} \mathrm{O}_{4} / \mathrm{Co}_{x} \mathrm{Zn}_{1-x} \mathrm{Fe}_{2} \mathrm{O}_{4}$ nanoparticles. ${ }^{41}$ However, much more research is required to suggest any association of nanoscale effects with such conception of symmetry and perfection, which is met in many instances of nature, science, and human art. 


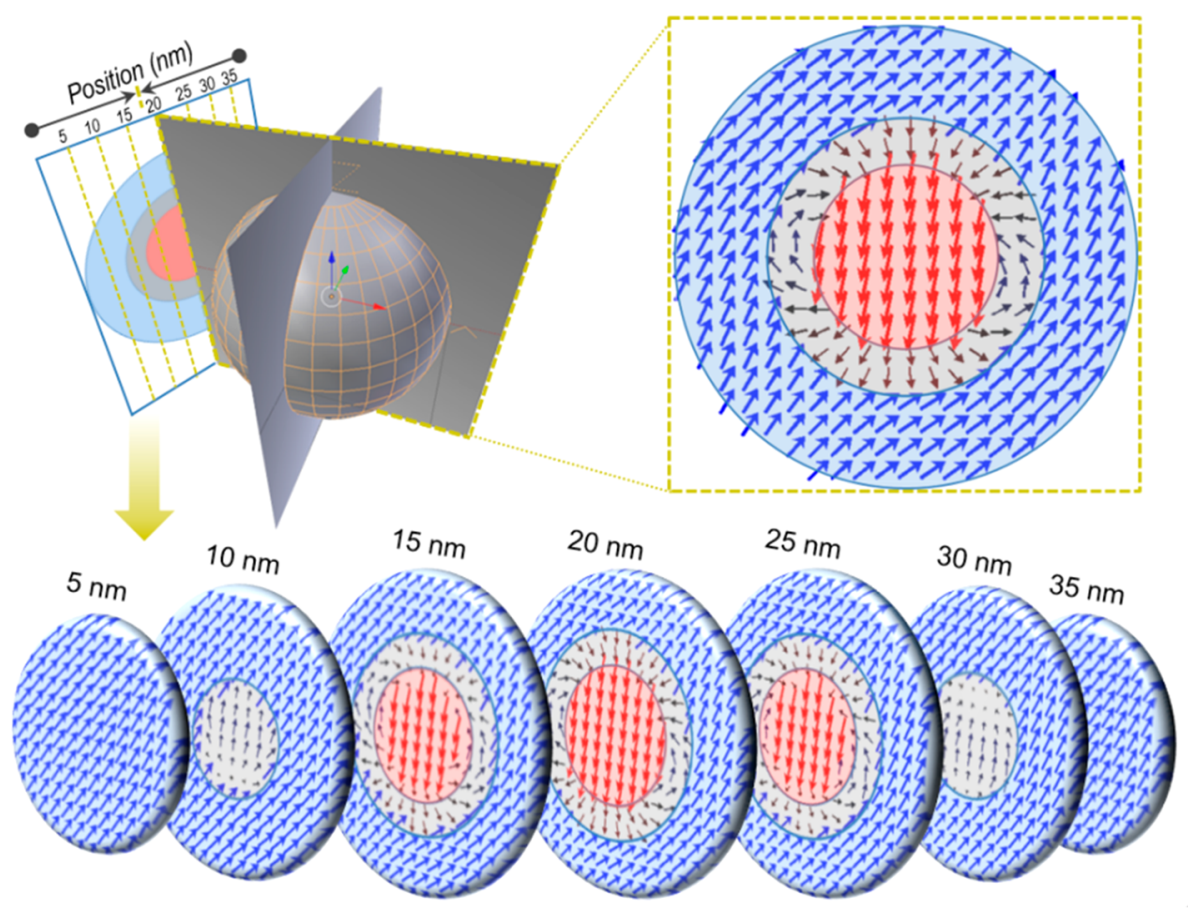

Figure 5. Micromagnetic magnetization configuration at remanence of an example core-shell structure $\left(D_{\text {tot }}=40 \mathrm{~nm} ; r_{\mathrm{C}}=16 \mathrm{~nm}, t_{\mathrm{sp}}=4 \mathrm{~nm} ; t_{\mathrm{S}}=\right.$ $8 \mathrm{~nm}$ ), in this case showing AFM-like coupling between core and shell. The snapshots are taken at regular cross sections of the particle, as illustrated by the sketch shown up, left. For clarity purposes the arrows representing the magnetization are magnified (each arrow corresponds to 8 cubic subunits of $1 \mathrm{~nm}$ side each).

Magnetic Modeling. The simplest and most generally employed model of magnetic hyperthermia is the linear response theory of Rosensweig. ${ }^{42}$ For a given particle size, this gives a scaling of the heating with $f \cdot H^{2}$. The measurements given in Figure 4 clearly do not conform to this scaling. To explain the behavior of such complex systems one must use a model allowing the possibility of nonuniform reversal with the capability to introduce the detailed structure of the core-shell combination. In an effort to infer which are the main coreshell features determining the magnetic response (e.g., aspect ratio, total size, etc.) and to correlate those with the heat release, a theoretical approach based on a micromagnetic technique using the OOMMF software package ${ }^{24}$ was carried out. An illustrative example of the micromagnetic simulations is shown in Figure 5. The simulated structure has a Fe core of radius $r_{\mathrm{C}}$, a magnetite shell of thickness $t_{\mathrm{S}}$, and a $\mathrm{FeO}$ interlayer of width $t_{\mathrm{sp}}$ treated as paramagnetic. In some cases, the shell was considered polycrystalline with random easy axis distribution. Considering the relatively large dimensions of the particles, we have focused on one field-amplitude/ frequency case $H_{\max }=30 \mathrm{mT}$ and $f=765 \mathrm{kHz}$ for blocked entities.

On the basis of the experimental details reported in Table 2, some representative examples of computed hysteresis loops are summarized in Figure 6, illustrating the different roles of the particle size $D_{\text {tot }}$ (panel A), the core-shell $t_{\mathrm{S}} / r_{\mathrm{C}}$ ratio (B), thickness of the paramagnetic interlayer spacer (C), and average size of shell crystallites (D) on the magnetization response. Overall, the results suggest several key roles of these four parameters.

A. Total particle size: For small values of $D_{\text {tot }}$ the particle behaves as a large magnetic supermoment with coherent magnetization behavior, while increasing $D_{\text {tot }}$ leads to a noncoherent magnetization reversal and narrower hysteresis loops. Interestingly, the threshold between regimes, which corresponds to an enhanced loop area, occurs at the transition at which the exchange energy contribution is similar to the magnetostatic one.

B Core-shell ratio: For a given $D_{\text {tot }}$ magnetization switching evolves from a noncoherent behavior (of small hysteresis area values) to a fully coherent one (with associated large areas) with increasing the $t_{\mathrm{S}} / r_{\mathrm{C}}$ value. Interestingly, the transition occurs around $t_{\mathrm{S}} / r_{\mathrm{C}}=$ 1.

C Thickness of interlayer spacer: The presence of a PMlike interlayer allows antiferromagnetic-like (AFM), that is, antiparallel, coupling between the core and the shell through magnetostatic interactions, being the soft shell the dominating phase, that is, switching first and forcing the core to switch later and also polarizing the $\mathrm{FeO}$. In such cases, the hysteresis area may be significantly enlarged.

D Polycrystalline shell may lead to a complete change in the shape of the loop, from smooth to very abrupt (squared) that would be highly desired for enhancing the heat output. ${ }^{43,44}$ It is worth noting that, in this case, both $\mathrm{Fe}$ and $\mathrm{Fe}_{3} \mathrm{O}_{4}$ phases are coupled in parallel configuration and the magnetization reversal is essentially coherent. Another important characteristic of the presence of a polycrystalline shell is that it significantly influences the dependence of the magnetic response on the direction of field application. Simulations were performed on the basis of the average shell crystallite size estimated by XRD diagrams for the oxide phases given in Table $\mathrm{S} 2$.

The comparison between experimentally measured and theoretically predicted heating performance is shown in Figure 7. Very good agreement between experiment and theory is 


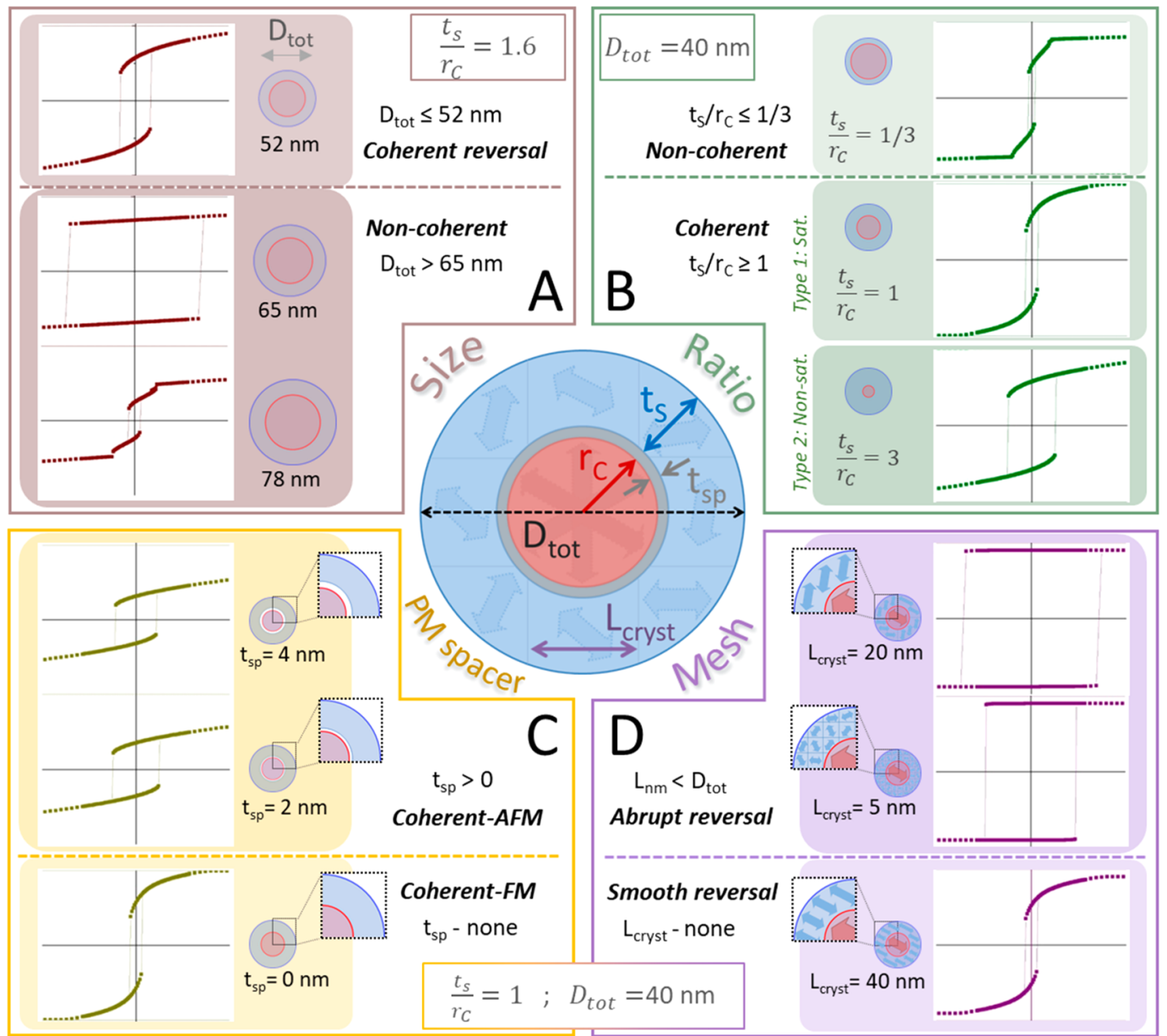

Figure 6. Case study of the hysteresis loops for an isolated particle depending on the total particle size (A), core-shell ratio (B), thickness $t_{s p}$ of the paramagnetic interlayer spacer (C), and average size $L_{\text {cryst }}$ of crystallites composing the shell (D). The central sketch depicts the associated coreshell structure.

obtained, except in the case of sample F03. Additional modeling (see the Supporting Information section S3) demonstrates that there is a crucial difference between sample F03 and rest: sample F03 is the only one not completely reversing the magnetization, that is, undergoing minor loop conditions-may this feature be enough to justify the divergence?

The implications of such minor-loop conditions regarding heating performance can be extremely important (minor loops correspond to much smaller heating power than the maximum achievable one; not to mention other effects such as e.g. higher dispersion in local heating performance ${ }^{28}$ ). However, the fact is that experimentally, the heat dissipated by this sample is not negligible, but comparable to those of samples F01 and F02 and even higher than that of F04. Clearly, there is significant heat dissipation from sample F03 that is not predicted by the theoretical model in the minor loop condition (see Figure S8). In the major loop conditions, even when a random distribution of nanoparticles is included, the predicted heat release is significantly higher than the experimentally measured value, as illustrated by the gray nonshaded tall column of sample F03 within Figure 7 (see Figure S11 and discussion therein).

To investigate this complex scenario, we have performed various modifications to the model, including the addition of perpendicular surface anisotropy in the outer layer of the shell or at the interface, dynamical effects, etc. The results, summarized in the Supporting Information section S4, indicate no improvement in the overall experiment-theory comparison but, on the contrary, increased divergence in some other samples. So, we are left with the conclusion that the presumed hypotheses stating that interparticle interactions plays a secondary role does not hold for sample F03, and that the dipole-dipole coupling changes the field parameters necessary to achieve the maximum $\mathrm{SAR}^{45}$ Our assumption is that nonreversing particles (as for example, those whose easy axes are almost parallel to the field direction) in the minor loop condition create a static field on the reversing particles. To test this, we next simulated the heating performance of pairs of potentially interacting particles from sample F03 at several separations and arrangements (see Figure S15). The results indicate a significant decrease of the heating performance with decreasing interparticle distances for special geometrical arrangements. We also show that in these situations the interparticle distances less than 1.5 times the particles' diameter can meaningfully explain the measured SAR values.

The reason why this effect of particle-particle interactions on SAR is only observed in sample F03 stems from the fact that this is the only sample exhibiting both requirements of coherent reversal and minor loop behavior.

Furthermore, Figure 8 presents magnetic configurations in three orthogonal planes for three samples F01, F02, and F04, which have similar sizes. The occurrence of noncoherent 


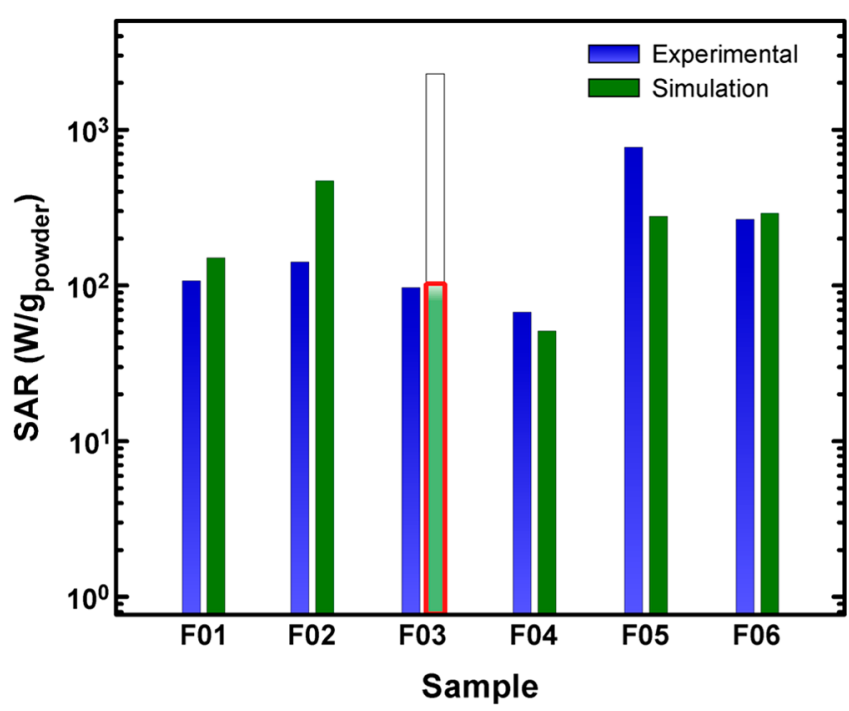

Figure 7. Comparison between experimentally measured SAR values (blue bars) and the theoretical predictions (green bars) obtained based on the model reported in Figure 6, for noninteracting conditions and field characteristics of $f=765 \mathrm{kHz}$ and $H_{\max }=30$ $\mathrm{mT}$. The SAR values are estimated from the area of the loops as SAR $=f$-area. Note that the computed heating efficiency for sample F03 describes the observational studies only if particle-particle dipolar coupling is included (marked red column), further explained in the text.

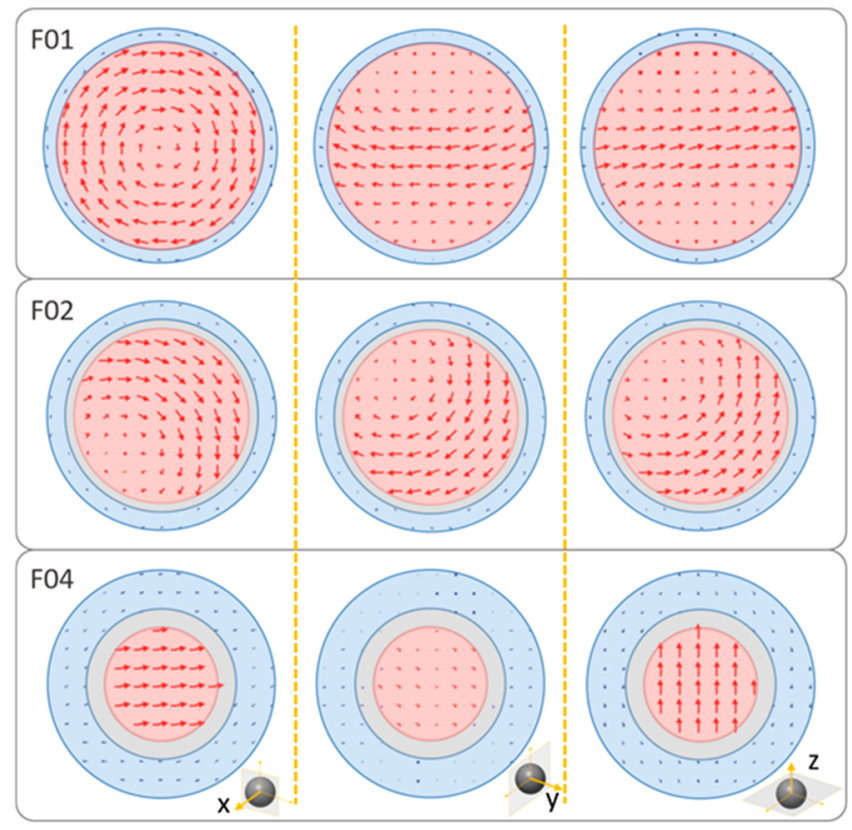

Figure 8. (A) Cross sections of magnetization configurations at remanence for the samples F01, F02, and F04 discussed in Table 2; the magnetization arrows correspond to the average over 8-unit cells. The snapshots have been taken along the $X, Y$, and $Z$ directions (left, center, and right snapshot for each sample, respectively) and always at the center of the particle.

reversal modes is clearly visible. Particularly, sample F01 demagnetizes through the occurrence of a $2 \mathrm{D}$ vortex, the core of which forms a tube, parallel to the easy-axis of $\mathrm{Fe}$ that is closer to the applied field direction. Sample F02 demagnetizes through a $3 \mathrm{D}$ vortex-like structure, in which the core is displaced from the center. Sample F04 presents almost coherent magnetization rotation. The occurrence of curling $2 \mathrm{D}$ or $3 \mathrm{D}$ vortices minimizes magnetostatic energy and diminishes the stray field of nanoparticles.

The above curved magnetic structures are topologically nontrivial. Recently, such structures in magnetism have attracted intensive research, given their potential applications to information technology. They have been also exploited by other researchers in the effort to fight cancer. For instance, the magnetic vortex in planar thin-film microdiscs creates an oscillation when a low frequency (tens of $\mathrm{Hz}$ ) AC magnetic field is applied. ${ }^{46}$ This movement transmits a mechanical force to the surrounding tissue that may be enough to compromise integrity of the cellular membrane. Additionally, coupling the vortex eigenmode to $\mathrm{MHz}$ frequencies may provide a new and efficient means of energy absorption by and emission from magnetic nanoparticles. ${ }^{47}$ However, from the standpoint of micromagnetism, our study differs completely from those not only because they were based on soft-magnetic permalloy, while our particles comprise a core and shell iron derivatives but also in the considered frequency range. Instead, our case resembles the one reported by Liu et $\mathrm{al}^{48}$ in iron oxide nanorings, in which magnetization is circumferential to the ring without stray fields. Minimization of stray fields is very crucial to reduce dipole-dipole interactions and agglomeration of magnetic particles in solution, supporting the argument that the oxide shell promotes decoupling of the iron nuclei. Such hypothesis was verified by establishing the effect of vortex-like intraparticle magnetization structures, FePt/iron oxide coreshell nanoparticles, in lowering of magnetic dipole-dipole interactions and enhancement of stabilization. ${ }^{49}$ And so, this work adds to the growing body of knowledge showing the complex issue of physical interpretation of interaction effects in magnetic hyperthermia. Finally, it is worth relating our work with the question posed by Z. Nemati et al., ${ }^{17}$ are core-shell magnetic nanoparticles promising for hyperthermia? Our answer is not only a clear yes, but furthermore, we emphasize the rich possibilities opened by tuning the core-shell coupling of those structures to tailor specific response.

\section{CONCLUSIONS}

In summary, distribution of iron and its oxides in well-defined core-shell nanoparticles is proven to be a crucial parameter when it comes to optimal magnetic properties in hyperthermia. This stems from the fact that the geometry of a coupled coreshell structure prohibits an existence of spatially homogeneous magnetization in the nanoparticle as whole, and when made of a magnetically softer-harder ferromagnets, for each thickness, there exists a critical radius where curling or more complicated nonhomogeneous structures become promoted. In particular, observations of topologically nontrivial vortex-like structures stress the difference between core-shell magnetic constructions and homogeneous particles.

Remarkably, in our case, samples with noncoherent reversal surprisingly seem more adequate in generating large SAR values because interparticle interactions are negligible in comparison with the inner magnetization processes, thus, diminishing the possibility of aggregated formation. Combined experimental and theoretical analysis also revealed a novel magnetization process wherein the iron-oxides effectively reduce the coercivity of the ferromagnetic cores by leading the magnetization process at small magnetic fields, thus, overcoming the main drawbacks of minor loops for stand-alone homogeneous nanoparticles for magnetoheating. Overall, we 
show the existence of far more options to tune the heating performance in magnetic nanoparticles as was foreseen up to now. The presented methodology can easily serve as flexible toolbox to support examination of nanoparticles behavior using different sizes, shapes, concentrations, mobilities, aggregation types, and dispersion media.

\section{ASSOCIATED CONTENT}

\section{S1 Supporting Information}

The Supporting Information is available free of charge at https://pubs.acs.org/doi/10.1021/acsanm.0c00568.

Further data on the analysis of particles morphology, the crystal structure, Mössbauer spectra, raw experimental observations on magnetic properties and hyperthermia efficiency and a deep explanation of the finite difference simulation method to theoretically derive the main core-shell features determining the magnetic response and correlate those with the heat release (PDF)

\section{AUTHOR INFORMATION}

\section{Corresponding Authors}

D. Serantes - Department of Physics, University of York, Heslington, York YO10 5DD, United Kingdom; Applied Physics Department and IIT, Universidade de Santiago de Compostela, Compostela 15782, Spain; 이이.org/0000-0002-3860-

2133; Email: david.serantes@usc.es

M. Angelakeris - Department of Physics, Aristotle University of Thessaloniki, Thessaloniki 54124, Greece; or orcid.org/00000001-9109-0221; Email: agelaker@auth.gr

\section{Authors}

K. Simeonidis - Department of Physics, Aristotle University of Thessaloniki, Thessaloniki 54124, Greece; Ecorecources P.C.,

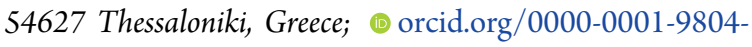
4690

C. Martinez-Boubeta - Freelancer in Bilbao, Bilbao 48007, Spain; orcid.org/0000-0001-7665-0649

S. Ruta - Department of Physics, University of York, Heslington, York YO10 5DD, United Kingdom

O. Chubykalo-Fesenko - Instituto de Ciencia de Materiales de Madrid, CSIC, Cantoblanco 28049, Spain; 자이.org/00000002-4081-1831

R. Chantrell - Department of Physics, University of York, Heslington, York YO10 5DD, United Kingdom; (1) orcid.org/ 0000-0001-5410-5615

J. Oró-Solé - Institut de Ciència de Materials de Barcelona, CSIC, Bellaterra 08193, Spain

Ll. Balcells - Institut de Ciencia de Materials de Barcelona, CSIC, Bellaterra 08193, Spain

A. S. Kamzin - Ioffe Physical-Technical Institute, Russian Academy of Sciences, St. Petersburg 194021, Russia

R. A. Nazipov - Kazan National Research Technological University, Kazan 420015, Russia

A. Makridis - Department of Physics, Aristotle University of Thessaloniki, Thessaloniki 54124, Greece; 이이. orcid.org/00000002-7609-1362

Complete contact information is available at: https://pubs.acs.org/10.1021/acsanm.0c00568

\section{Author Contributions}

The manuscript was written through contributions of all authors. All authors have given approval to the final version of the manuscript.

\section{Notes}

The authors declare no competing financial interest.

\section{ACKNOWLEDGMENTS}

Financial support of the Royal Society through International Exchanges scheme IE160535 is gratefully acknowledged. This work made use of computational facilities funded by the Small items of research equipment at the University of York ENERGY (Grant No. EP/K031589/1). We thank the CNRS-PROMES laboratory, UPR 8521, belonging to the French National Centre for Scientific Research (CNRS) for providing access to its installations, the support of its scientific and technical staff (N. Boullet, E. Guillot, A. Badziaka), and the financial support of the SFERA-III project (Grant Agreement No 823802). We also acknowledge the Centro de Supercomputación de Galicia (CESGA) for the computational resources. This research was partially supported by the Conselleria de Educación Program for Development of a Strategic Grouping in Materials (AeMAT) at the Universidade de Santiago de Compostela (Grant No. ED431E2018/08, Xunta de Galicia). D.S. also acknowledges Xunta de Galicia for financial support under the I2C Plan. K.S. acknowledges Stavros Niarchos Foundation for financial support. C.M.B. would like to thank his wife, Marian, for sustaining him during the many rough draft versions of this paper.

\section{REFERENCES}

(1) Houlding, T. K.; Rebrov, E. V. Application of Alternative Energy Forms in Catalytic Reactor Engineering. Green Process. Synth. 2012, DOI: 10.1515/greenps-2011-0502.

(2) Beck, M. M.; Lammel, C.; Gleich, B. Improving Heat Generation of Magnetic Nanoparticles by Pre-Orientation of Particles in a Static Three Tesla Magnetic Field. J. Magn. Magn. Mater. 2017, 427, 195.

(3) Colombo, M.; Carregal-Romero, S.; Casula, M. F.; Gutiérrez, L.; Morales, M. P.; Böhm, I. B.; Heverhagen, J. T.; Prosperi, D.; Parak, W. J. Biological Applications of Magnetic Nanoparticles. Chem. Soc. Rev. 2012, 41 (11), 4306.

(4) Angelakeris, M. Magnetic Nanoparticles: A Multifunctional Vehicle for Modern Theranostics. Biochim. Biophys. Acta, Gen. Subj. 2017, 1861, 1642-1651.

(5) Lanier, O. L.; Monsalve, A. G.; McFetridge, P. S.; Dobson, J. Magnetically Triggered Release of Biologics. Int. Mater. Rev. 2019, 64, 63.

(6) PéRigo, E. A.; Hemery, G.; Sandre, O.; Ortega, D.; Garaio, E.; Plazaola, F.; Teran, F. J. Fundamentals and Advances in Magnetic Hyperthermia. Appl. Phys. Rev. 2015, 2, 041302.

(7) Yagawa, Y.; Tanigawa, K.; Kobayashi, Y.; Yamamoto, M. Cancer Immunity and Therapy Using Hyperthermia with Immunotherapy, Radiotherapy, Chemotherapy, and Surgery. J. Cancer Metastasis Treat. 2017, 3 (10), 218.

(8) Ranoo, S.; Lahiri, B. B.; Muthukumaran, T.; Philip, J. Enhancement in Hyperthermia Efficiency under in Situ Orientation of Superparamagnetic Iron Oxide Nanoparticles in Dispersions. Appl. Phys. Lett. 2019, 115, 043102.

(9) Conde-Leboran, I.; Baldomir, D.; Martinez-Boubeta, C.; Chubykalo-Fesenko, O.; Del Puerto Morales, M.; Salas, G.; Cabrera, D.; Camarero, J.; Teran, F. J.; Serantes, D. A Single Picture Explains Diversity of Hyperthermia Response of Magnetic Nanoparticles. J. Phys. Chem. C 2015, 119, 15698.

(10) Sun, J.; Fan, F.; Wang, P.; Ma, S.; Song, L.; Gu, N. OrientationDependent Thermogenesis of Assembled Magnetic Nanoparticles in 
the Presence of an Alternating Magnetic Field. ChemPhysChem 2016, 17, 3377.

(11) Sanz, B.; Calatayud, M. P.; Cassinelli, N.; Ibarra, M. R.; Goya, G. F. Long-Term Stability and Reproducibility of Magnetic Colloids Are Key Issues for Steady Values of Specific Power Absorption over Time. Eur. J. Inorg. Chem. 2015, 2015, 4524.

(12) Bellizzi, G.; Bucci, O. M.; Chirico, G. Numerical Assessment of a Criterion for the Optimal Choice of the Operative Conditions in Magnetic Nanoparticle Hyperthermia on a Realistic Model of the Human Head. Int. J. Hyperthermia 2016, 32, 688.

(13) Serantes, D.; Simeonidis, K.; Angelakeris, M.; ChubykaloFesenko, O.; Marciello, M.; del Puerto Morales, M.; Baldomir, D.; Martinez-Boubeta, C. Multiplying Magnetic Hyperthermia Response by Nanoparticle Assembling. J. Phys. Chem. C 2014, 118, 5927.

(14) Eapen, J.; Rusconi, R.; Piazza, R.; Yip, S. The Classical Nature of Thermal Conduction in Nanofluids. J. Heat Transfer 2010, DOI: $10.1115 / 1.4001304$.

(15) Di Corato, R.; Espinosa, A.; Lartigue, L.; Tharaud, M.; Chat, S.; Pellegrino, T.; Ménager, C.; Gazeau, F.; Wilhelm, C. Magnetic Hyperthermia Efficiency in the Cellular Environment Fordifferent Nanoparticle Designs. Biomaterials 2014, 35, 6400.

(16) Dutz, S.; Hergt, R. Magnetic Particle Hyperthermia - A Promising Tumour Therapy? Nanotechnology 2014, 25, 452001.

(17) Nemati, Z.; Alonso, J.; Khurshid, H.; Phan, M. H.; Srikanth, H. Core/Shell Iron/Iron Oxide Nanoparticles: Are They Promising for Magnetic Hyperthermia? RSC Adv. 2016, 6 (45), 38697-38702.

(18) Zhang, Q.; Castellanos-Rubio, I.; Munshi, R.; Orue, I.; Pelaz, B.; Gries, K. I.; Parak, W. J.; Del Pino, P.; Pralle, A. Model Driven Optimization of Magnetic Anisotropy of Exchange-Coupled CoreShell Ferrite Nanoparticles for Maximal Hysteretic Loss. Chem. Mater. 2015, 27, 7380 .

(19) Martinez-Boubeta, C.; Simeonidis, K.; Amarantidis, S.; Angelakeris, M.; Balcells, L.; Monty, C. Scaling up the Production of Magnetic Nanoparticles for Biomedical Applications: CostEffective Fabrication from Basalts. Phys. Status Solidi Curr. Top. Solid State Phys. 2014, 11 (5-6), 1053-1058.

(20) Semenov, V. G.; Panchuk, V. V. Mössbauer Spectra Processing Program MossFit. Private Communication.

(21) Chalkidou, A.; Simeonidis, K.; Angelakeris, M.; Samaras, T.; Martinez-Boubeta, C.; Balcells, L.; Papazisis, K.; Dendrinou-Samara, C.; Kalogirou, O. In Vitro Application of $\mathrm{Fe} / \mathrm{MgO}$ Nanoparticles as Magnetically Mediated Hyperthermia Agents for Cancer Treatment. J. Magn. Magn. Mater. 2011, 323, 775.

(22) Simeonidis, K.; Martinez-Boubeta, C.; Balcells, L.; Monty, C.; Stavropoulos, G.; Mitrakas, M.; Matsakidou, A.; Vourlias, G.; Angelakeris, M. Fe-Based Nanoparticles as Tunable Magnetic Particle Hyperthermia Agents. J. Appl. Phys. 2013, 114 (10), 103904.

(23) Makridis, A.; Curto, S.; Van Rhoon, G. C.; Samaras, T.; Angelakeris, M. A Standardisation Protocol for Accurate Evaluation of Specific Loss Power in Magnetic Hyperthermia. J. Phys. D: Appl. Phys. 2019, 52, 255001.

(24) Donahue, M.; Porter, D. OOMMF User's Guide, vers. 1.0, Interag. Rep. NISTIR 6376; NIST: Gaithersburg, MD, 1999.

(25) Simeonidis, K.; Morales, M. P.; Marciello, M.; Angelakeris, M.; De La Presa, P.; Lazaro-Carrillo, A.; Tabero, A.; Villanueva, A.; Chubykalo-Fesenko, O.; Serantes, D. In-Situ Particles Reorientation during Magnetic Hyperthermia Application: Shape Matters Twice. Sci. Rep. 2016, DOI: 10.1038/srep38382.

(26) Ruta, S.; Chantrell, R.; Hovorka, O. Unified Model of Hyperthermia via Hysteresis Heating in Systems of Interacting Magnetic Nanoparticles. Sci. Rep. 2015, DOI: 10.1038/srep09090.

(27) Serantes, D.; Baldomir, D.; Martinez-Boubeta, C.; Simeonidis, K.; Angelakeris, M.; Natividad, E.; Castro, M.; Mediano, A.; Chen, D. X.; Sanchez, A.; et al.. Influence of Dipolar Interactions on Hyperthermia Properties of Ferromagnetic Particles. J. Appl. Phys. 2010, 108, 073918.

(28) Munoz-Menendez, C.; Serantes, D.; Ruso, J. M.; Baldomir, D. Towards Improved Magnetic Fluid Hyperthermia: Major-Loops to
Diminish Variations in Local Heating. Phys. Chem. Chem. Phys. 2017, $19,14527$.

(29) Martinez-Boubeta, C.; Simeonidis, K.; Serantes, D.; CondeLeborán, I.; Kazakis, I.; Stefanou, G.; Peña, L.; Galceran, R.; Balcells, L.; Monty, C. Adjustable Hyperthermia Response of Self-Assembled Ferromagnetic Fe-MgO Core-Shell Nanoparticles by Tuning DipoleDipole Interactions. Adv. Funct. Mater. 2012, 22, 3737.

(30) Martínez-Boubeta, C.; Simeonidis, K.; Angelakeris, M.; PazosPérez, N.; Giersig, M.; Delimitis, A.; Nalbandian, L.; Alexandrakis, V.; Niarchos, D. Critical Radius for Exchange Bias in Naturally Oxidized Fe Nanoparticles. Phys. Rev. B: Condens. Matter Mater. Phys. 2006, DOI: 10.1103/PhysRevB.74.054430.

(31) Baaziz, W.; Pichon, B. P.; Fleutot, S.; Liu, Y.; Lefevre, C.; Greneche, J. M.; Toumi, M.; Mhiri, T.; Begin-Colin, S. Magnetic Iron Oxide Nanoparticles: Reproducible Tuning of the Size and Nanosized-Dependent Composition, Defects, and Spin Canting. J. Phys. Chem. C 2014, 118, 3795.

(32) Redl, F. X.; Black, C. T.; Papaefthymiou, G. C.; Sandstrom, R. L.; Yin, M.; Zeng, H.; Murray, C. B.; O’Brien, S. P. Magnetic, Electronic, and Structural Characterization of Nonstoichiometric Iron Oxides at the Nanoscale. J. Am. Chem. Soc. 2004, 126, 14583.

(33) Sinković, B.; Johnson, P. D.; Brookes, N. B.; Clarke, A.; Smith, N. V. Magnetic Structure of Oxidized $\mathrm{Fe}(001)$. Phys. Rev. Lett. 1990, 65 (13), 1647-1650.

(34) Roosendaal, S. J.; Vredenberg, A. M.; Habraken, F. H. P. M. Oxidation of Iron: The Relation between Oxidation Kinetics and Oxide Electronic Structure. Phys. Rev. Lett. 2000, 84, 3366.

(35) Estrader, M.; López-Ortega, A.; Golosovsky, I. V.; Estradé, S.; Roca, A. G.; Salazar-Alvarez, G.; López-Conesa, L.; Tobia, D.; Winkler, E.; Ardisson, J. D. Origin of the Large Dispersion of Magnetic Properties in Nanostructured Oxides: FexO/Fe3O4 Nanoparticles as a Case Study. Nanoscale 2015, 7, 3002.

(36) Panicaud, B.; Grosseau-Poussard, J. L.; Dinhut, J. F. On the Growth Strain Origin and Stress Evolution Prediction during Oxidation of Metals. Appl. Surf. Sci. 2006, 252, 5700.

(37) Stoner, A. E. C.; Wohlfarth, E. P. A Mechanism of Magnetic Hysteresis in Heterogeneous Alloys Source. Philosophical Transactions of the Royal Society of London. Series A, Mathematical and Physical Sciences 1948, 240 (826), 599-642.

(38) Kaur, M.; McCloy, J. S.; Jiang, W.; Yao, Q.; Qiang, Y. Size Dependence of Inter- and Intracluster Interactions in Core-Shell IronIron Oxide Nanoclusters. J. Phys. Chem. C 2012, 116, 12875.

(39) Bakoglidis, K. D.; Simeonidis, K.; Sakellari, D.; Stefanou, G.; Angelakeris, M. Size-Dependent Mechanisms in AC Magnetic Hyperthermia Response of Iron-Oxide Nanoparticles. IEEE Trans. Magn. 2012, 48, 1320.

(40) Balivada, S.; Rachakatla, R. S.; Wang, H.; Samarakoon, T. N.; Dani, R. K.; Pyle, M.; Kroh, F. O.; Walker, B.; Leaym, X.; Koper, O. B.; et al. A/C Magnetic Hyperthermia of Melanoma Mediated by Iron(0)/Iron Oxide Core/Shell Magnetic Nanoparticles: A Mouse Study. BMC Cancer 2010, DOI: 10.1186/1471-2407-10-119.

(41) Lavorato, G. C.; Das, R.; Xing, Y.; Robles, J.; Litterst, F. J.; Baggio-Saitovitch, E.; Phan, M.-H.; Srikanth, H. Origin and ShellDriven Optimization of the Heating Power in Core/Shell Bimagnetic Nanoparticles. ACS Appl. Nano Mater. 2020, 3, 1755.

(42) Rosensweig, R. E. Heating Magnetic Fluid with Alternating Magnetic Field. J. Magn. Magn. Mater. 2002, 252, 370.

(43) Conde-Leborán, I.; Serantes, D.; Baldomir, D. Orientation of the Magnetization Easy Axes of Interacting Nanoparticles: Influence on the Hyperthermia Properties. J. Magn. Magn. Mater. 2015, 380, 321.

(44) Das, R.; Witanachchi, C.; Nemati, Z.; Kalappattil, V.; Rodrigo, I.; García, J. Á.; Garaio, E.; Alonso, J.; Lam, V. D.; Le, A.-T.; et al. Magnetic Vortex and Hyperthermia Suppression in Multigrain Iron Oxide Nanorings. Appl. Sci. 2020, 10 (3), 787.

(45) Kashevsky, B. E.; Kashevsky, S. B.; Zholud, A. M. Magnetic Hyperthermia with Hard-Magnetic Particles: Solid and Liquid Systems of Paired Stoner-Wohlfarth Particles. J. Magn. Magn. Mater. 2019, 477, 307. 
(46) Kim, D. H.; Rozhkova, E. A.; Ulasov, I. V.; Bader, S. D.; Rajh, T.; Lesniak, M. S.; Novosad, V. Biofunctionalized Magnetic-Vortex Microdiscs for Targeted Cancer-Cell Destruction. Nat. Mater. 2010, 9, 165.

(47) Kim, S. K.; Yoo, M. W.; Lee, J.; Lee, J. H.; Kim, M. K. Resonant Vortex-Core Reversal in Magnetic Nano-Spheres as Robust Mechanism of Efficient Energy Absorption and Emission. Sci. Rep. 2016, DOI: $10.1038 /$ srep31513.

(48) Liu, X. L.; Yang, Y.; Ng, C. T.; Zhao, L. Y.; Zhang, Y.; Bay, B. H.; Fan, H. M.; Ding, J. Magnetic Vortex Nanorings: A New Class of Hyperthermia Agent for Highly Efficient in Vivo Regression of Tumors. Adv. Mater. 2015, 27, 1939.

(49) Yang, M. Da; Ho, C. H.; Ruta, S.; Chantrell, R.; Krycka, K.; Hovorka, O.; Chen, F. R.; Lai, P. S.; Lai, C. H. Magnetic Interaction of Multifunctional Core-Shell Nanoparticles for Highly Effective Theranostics. Adv. Mater. 2018, 30, 1802444. 